NAL PROPOSAL NO. 153

Scientific spokesman:

Toshio Kitagaki

Bubble Chamber Analysis Center

Department of Physics

Tohoku University

Katahira 2-chome,

Sendai, Japan

Tel. (0222) 22-1828 (Ext. 4428)

NAL PROPOSAL (30" HBC)

Negative Pion - Proton Interactions at $200 \mathrm{GeV} / \mathrm{C}$

Tohoku University Group

T. Kitagaki, S. Tanaka, K. Abe, K. Hasegawa,

R. Sugahara, K. Tamai, H. Kichimi,

T. Okus awa and S. Noguchi

July 1971 
NAL PROPOSAL $\left(30^{\prime \prime} \cdot \mathrm{HBC}\right)$

Title: Negative Pion - Proton Interactions at $200 \mathrm{GeV} / \mathrm{c}$

Experimenters: . Tohoku University Group

T. Kitagalki, S. Tanaka, K. Abe, K. Hasegawa,

R. Sugahara, K. Tamai, H. Kichimi,

T. Okusawa and S. Noguchi

We will add other collaborators later inside Japan.

Correspondent: Toshio Kitagaki, Profossor

Bubble Chamber Analysis Center,

Department of Physics,

Tohoku University, .

Katahira 2-chome,

Sendai, Japan

Tel. (0222) 22-1828 (Ext. 4428)

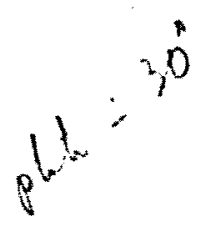

(July 1971) 
I. We propose here to take 50,000 pictures of the NAL 30-inch bubble chamber, filled with hydrogen and exposed to $200 \mathrm{GeV} / \mathrm{c}$ beams of negative pions.

We intend to do.study the technical limitations of the use of a bubble chamber for such high energy interactions. At the same time we will investigate the physics contained in the pictures as much as and as soon as possible. We expect to finish the study made only by scanning in a month and to end all studies in six months after getting film. Even in the energy of $200 \mathrm{GeV}$ region, interactions will be accompanied with some slow secondary particles, which will be the object of analysis most interest. However majority of interactions may have a jet like topology. We will mainly use our FSD for the analysis of this experiment and the accuracy in momentum measurement and the separation of each track in a condenced jet may be the main subjects of our technical study.

In the 30-inch bubble chamber, we propose to insert a target plate and a gamma conversion plate, if the situation of the chamber will allow to do so. A short discussion for the insertion of plates will be shown later. 
II. For the physics, we reasonably intend to investigate as much as possible in a short period. The discussed subjects in our group are presently as follows.

a). Study of the charged particle multiplicity at $200 \mathrm{GeV} / \mathrm{c}$ in $\pi^{-}-p$ interactions. Various models have been suggested for the description on this matter; the multiperipheral model, the difractive model based on the Regge pole theory and the others. On the other hand, the available experimental data in $\pi^{-}-p$ interactions at higher energy than $30 \mathrm{GeV} / \mathrm{c}$ are seldom. We plan to do make scanning and rescanning carefully. The scanning in a month period will produce an important data with high statistics. Our original proposal with gamma conversion plate will add an information about the neutral pion multiplicity.

b). The accurate measurement of the total cross section in $\pi^{-}-p$ interactions. For determining total cross section accurately, the recoil protons of the 2-prong events will be analyzed, and the scanning loss due to short tracks will be corrected.

c). Inclusive studies for pions. These days the study of the inclusive type is becoming important; for example a scaling limit and so on. We will investigate the inclusive reactions,

$$
\begin{aligned}
\pi^{-}+p & \rightarrow \pi^{-}+\text {anything } \\
& \rightarrow \pi^{+}+\text {anything }
\end{aligned}
$$

Low momentum secondaries $(\lesssim 1 \mathrm{GeV} / \mathrm{c})$ will have special roll in this study. The number of available events with the slow 
secondaries will be approximately 10,000 .

d). The cross section of the reactions

$$
\pi^{-}+\mathrm{p} \longrightarrow \mathrm{Y}^{\mathrm{o}}+\text { anything. }
$$

The number of the vee events is estimated as approximately 70. Contamination from the $\mathrm{K}^{\circ}$ decay is negligibly small. We will make a study of the inclusive type in this reaction, too.

e) We also analyse ali the events by the conventional methods used in the cosmic ray research, especially for jet with high momentum tracks. This type of analysis will, at the same time, give an important check against the method in the cosmic ray research. 
III. If the conditions of the chamber permit, we make the following requirement for our experiment. A target plate and a gamma conversion plate are inserted in the chamber and located as shown in Fig. 1. The target plate is made of Aluminum with $1.2 \mathrm{gr}$. thickness which is equivalent to our fiducial length of $20 \mathrm{~cm}$ in the liquid hydrogen. The gamma conversion plate is Tantalum of two radiation length. The merits of inserting these plates in the chamber are;

1. to increase the target volume and to have a long measurable length of tracks.

2. to compare the data between from the liquid hydrogen and from the Al-plate.

3. to detect the outgoing neutral pions by Ta-plate.

IV. It is estimated that the average number of incident track 6 per picture is adequate in the exposure with the target plate. The event-rate is 0.14 events per picture in the 20 $\mathrm{cm}$ liquid hydrogen of the fiducial volume and 0.07 in the aluminum target plate. However, if the plates is not able to be inserted in the chamber, we request the average 8 -incident tracks per picture. 
The expected number of events are by topology;

$$
\text { in liq. } \mathrm{H}_{2} \text { in Al-Plate }
$$

\begin{tabular}{lrr}
2 -prong & 500 & 250 \\
4 -prong & 1100 & 550 \\
6 -prong & 1400 & 700 \\
8 -prong & 1300 & 650 \\
10 -prong & 700 & 350 \\
12 -prong & 500 & 250 \\
$14-$ prong & 300 & 150 \\
16 -prong & 100 & 50 \\
\hline & & 35 \\
Vee-associated & 70 & \\
events & & \\
\hline
\end{tabular}

In the estimation, the following parameters are used;

$$
\begin{array}{lc}
\text { fiducial length } & 20 \mathrm{~cm}, \\
\text { incident beam } & 6 \text { pions/picture, } \\
\text { half of the beam entrance in the chamber is } \\
\text { covered by Al-plate with } 1.2 \mathrm{sr} \text { thickness. } \\
\text { (see Fig. 1) }
\end{array}
$$

V. Exposure is desired as soon as possible. Four members of our group will be sent from Japan to NAL to attend the exposure. They will stay there for about a month including the exposure period. 


\section{Measurement and Analysis}

Our staffs and facilities in July 1971 are;

a) Manpower in the Toholu University Group is,

1 Professor

2 Associate professors

4 Research associates

3 Technicians

5 Graduate students

10 scanners

b) Four scanning projectors are in operation.

c) Six digitizers, for three role film of $70 / 35 \mathrm{~mm}$, are in operation. Four specially designed digitizers, for four role film of $70 / 35^{\circ} \mathrm{mm}$, are under construction.

d) Three-view flying spot digitizers are under adjustment and the single view operation will start by the end of 1971.

e) The following computers are used for analysis; TOSBAC 3400 model 41 (Bubble chamber analysis center, IBM 7090 equivalent).

NEAC 2200 model 700 (Tohoku university computer center, IBM $7090 \times 15$ equivalent) 


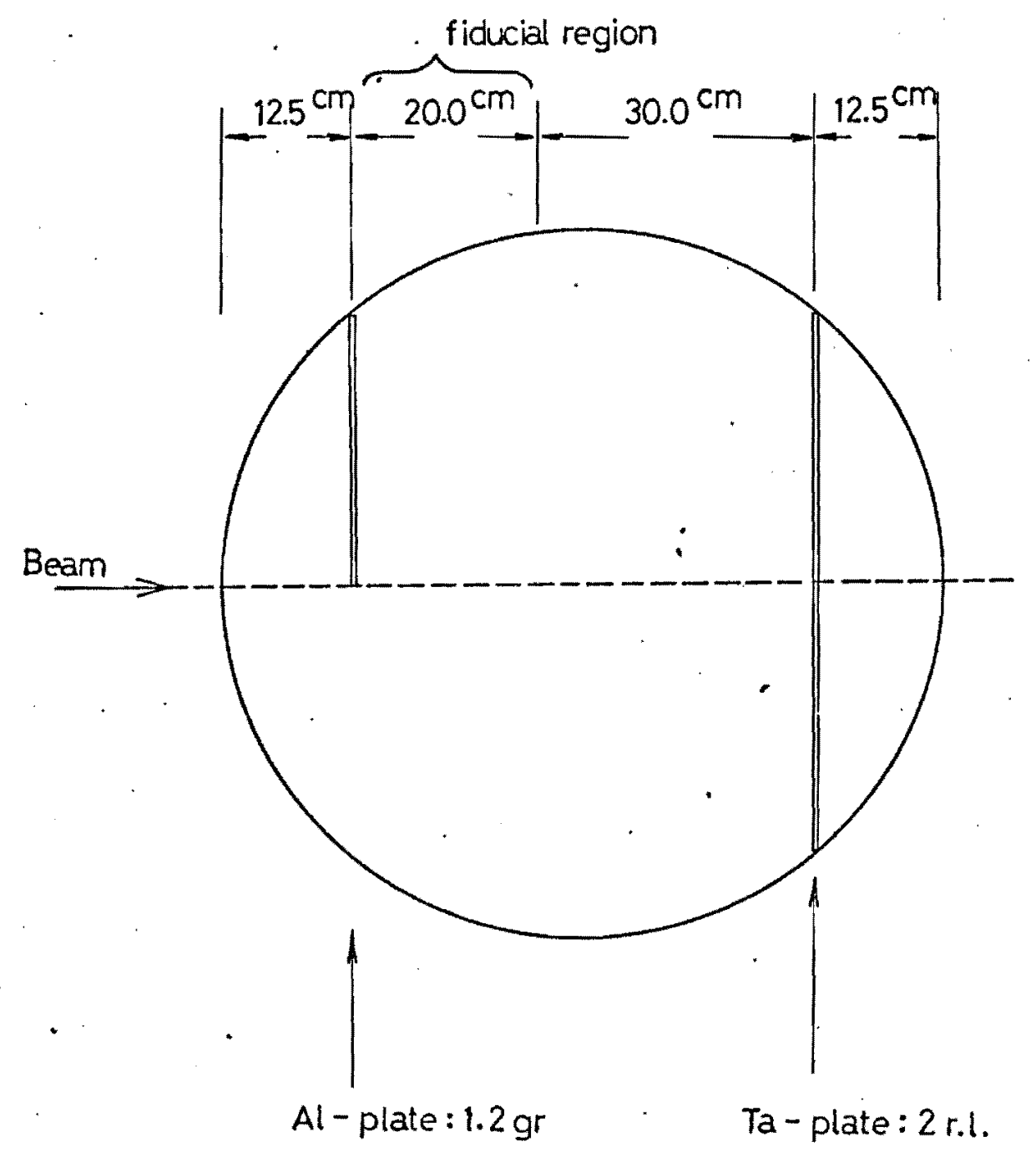

Fig.1. plates in the 30 -inch chamber 